Prospective Evaluation

\title{
Pulsed Compared to Thermal Radiofrequency to the Medial Calcaneal Nerve for Management of Chronic Refractory Plantar Fasciitis: A Prospective Comparative Study
}

Ayman M. Osman, MD'1, Dina H. El-Hammady, MD², and Mohamed M. Kotb, MD³

From: ${ }^{2}$ Assistant professor of Anesthesiology, Intensive Care and Pain Medicine, Assiut University; ${ }^{2}$ Assistant professor of Rheumatology and Rehabilitation, Faculty of

Medicine, Assiut University ${ }^{3}$ Assistant professor of Upper limb, Hand and Reconstructive Microsurgery Unit, Orthopedic Department, Faculty of Medicine, Assiut University

Address Correspondence: Dina H. el-Hammady, MD Assiut University Faculty of Medicine Assiut, Egypt E-mail:

dinael_hammady73@yahoo.com

Disclaimer: There was no external funding in the preparation of this manuscript. Conflict of interest: Each author certifies that he or she, or a member of his or her immediate

family, has no commercial association (i.e., consultancies, stock ownership, equity interest, patent/licensing arrangements, etc.) that might pose a conflict of interest in connection with the submitted manuscript.

Manuscript received: 02-09-2016 Revised manuscript received: 04-23-2016

Accepted for publication: 05-16-2016

Free full manuscript: www.painphysicianjournal.com
Background: Radiofrequency (RF) treatment is a minimally invasive procedure that has been used for more than 3 decades in treating various chronic pain syndromes. Conventional (continuous) RF treatment occasionally results in worsening or even initiating a new type of pain. The use of pulsed radiofrequency (PRF), which has a non- or minimally neurodestructive neuromodulatory effect, serves as an alternative to conventional RF therapy in many medical situations.

Objectives: To evaluate the effect of applying PRF for 6 minutes vs. thermal radiofrequency (TRF) for 90 seconds to the medial calcaneal nerve for treatment of chronic refractory plantar fasciitis pain.

Study Design: Prospective comparative study.

Setting: Pain, Orthopedic, and Rheumatology and Rehabilitation Clinics of Assiut University Hospital.

Methods: Twenty patients with refractory chronic bilateral plantar fasciitis received PRF to the medial calcaneal nerve for 6 minutes for one heel and TRF to the same nerve on the other heel (as their own control) for 90 seconds. Numerical verbal rating scale (NVRS) at waking up from bed and after prolonged walking, and satisfaction score were used for assessment of studied patients at one, 3, 6, 12, and 24 weeks from the intervention.

Results: All studied patients showed significant improvement in their pain scale after the intervention that lasted for 24 weeks; however, the PRF heels had significantly better pain scale and satisfaction scores at the first and third weeks assessments when compared to the TRF heels. Effective analgesia was achieved after one week or less after PRF compared to 3 weeks for the TRF $(P<0.001)$.

Limitations: No randomization.

Conclusions: PRF to the medial calcaneal nerve is a safe and effective method for treatment of chronic plantar fasciitis pain. The onset of effective analgesia can be achieved more rapidly with PRF compared to TRF on the same nerve. Further randomized trials are needed to confirm the therapeutic effect and optimizing the dose of RF needed.

Key words: Pulsed radiofrequency, thermal radiofrequency, medial calcaneal nerve, plantar fasciitis, plantar aponeurosis, visual analogue scale

Pain Physician 2016; 19:E1181-E1187 
lantar fasciitis is an inflammatory condition of the plantar fascia which is very painful and disabling (1). Bilateral involvement of plantar fasciitis occurs in 20 to $30 \%$ of patients (2). The exact etiology of plantar fasciitis is unclear. The pathogenesis of plantar fasciits involves physical-mechanical overload and micro tears within the fascia $(3,4)$. This pathogenesis involves localized inflammation and degeneration of the proximal plantar aponeurosis, near its origin (medial tuberosity of the calcaneum) (1). The pain associated with plantar fasciitis has been attributed to inflammation in the area where the plantar fascia attaches near the anteromedial tubercle of the calcaneus. Other hypothesis may include fractured bony heel spur, chronic tears in the plantar fascia, or nerve entrapment (5).

Plantar fasciitis pain is usually presented by medial plantar heel pain, especially during the first weightbearing steps in the morning or after prolonged periods of rest (4). Although the pain may subside with activity, in some patients it may persist, interrupting activities of daily living. Initial reports of the heel pain may be diffuse or migratory; however, with time, it usually focuses around the area of the medial calcaneal tuberosity (6).

Physical examination presents with localized tenderness at the antero-medial aspect of the calcaneus, and on palpation, the entire plantar surface is tender and located more into the arch in true plantar fasciitis as compared to heel pain syndrome in which the maximum tenderness is just anterior to the calcaneal tuberosity or at the plantar medial heel. Pain may be exacerbated by passive dorsiflexion of the toes or having the patient stand on the tips of the toes $(7,8)$.

The treatment of plantar fasciitis includes operative and non-operative management with no single treatment proven to be the most beneficial $(1,3)$. Conservative therapy of plantar fasciitis is successful in the vast majority of cases. Rest or minimization of running or jumping is the cornerstone of therapy. Heel cups are sometimes helpful in alleviating symptoms, presumably by padding the heel and absorbing the impact of walking. Nonsteroidal anti-inflammatory drugs are also helpful in acute cases. Extracorporeal shock wave therapy may also be of benefit. If the above measures are ineffective, local injection of steroids may be effective $(4,5)$. Improvement is frustratingly slow and gradual, taking up to a year in some cases. For refractory cases, either open or endoscopic plantar fasciotomy may be considered (5).
Thermal radiofrequency (TRF) has been proposed as another method to treat chronic heel pain. The heat partially damages the nerve and stops pain transmission. The proposed mechanism of action is desensitization of the nerve endings (3). Recent evidence suggests that the electric field, rather than the heat lesion, is responsible for the clinical effect of radiofrequency (RF) (9).

Pulsed radiofrequency (PRF) is a recent non-neurodestructive method that provides pain relief (10). The relatively long pause between pulses allows for heat dissipation, principally through conduction and convection. This results in a temperature in the surrounding tissue that is insufficient to produce neural coagulation (11). PRF nerve lesioning is a safe intervention (12). The risks of neuritis, deafferentation pain, and neuroma formation are minimal when compared to TRF (9).

Although there are scarce data on using RF to the calcaneal nerve in treatment of plantar fasciitis (13), TRF to the calcaneal nerve was proven to improve plantar fasciitis pain in the literature $(13,14)$. On the other hand, there have been 2 case reports describing the success of PRF in the treatment of refractory plantar fasciitis. However, these studies never recorded patient improvement in the first month and the actual onset of effective analgesia was never traced before one month.

The current study aims to compare the effect of applying PRF versus TRF on the medial calcaneal nerve for treatment of chronic refractory plantar fasciitis.

\section{Methods}

The current work was a prospective non-randomized comparative study. Patients were recruited from the outpatient pain clinic, the Orthopedic Clinic and Rehabilitation Clinic of Assiut University Hospital during the period from January 1 to the end of December 2015. Twenty patients with bilateral chronic plantar fasciitis (> 6 months) refractory to medical treatment (including silicon heel cup, oral anti-inflammatory, and one local steroid injection) were included in the study. Detailed clinical history and examination was the mainstay for diagnosis. Moreover, patients with other causes of heel pain were excluded, including calcaneal stress fracture, Achilles tendinopathy, and tarsal tunnel syndrome. $\mathrm{X}$-ray, magnetic resonance imaging (MRI), and nerve conduction studies were ordered as required (5). All patients signed a written consent form after reading the patient information sheet describing the procedure, its potential benefits, and possible hazards. The difference between modes of treatment had been explained to all 
participants. The study protocol had been approved by the Institutional Ethical Review Board.

Medial calcaneal nerve block using $2 \mathrm{~mL}$ lidocaine $2 \%$ was performed bilaterally in all patients one week before the intervention. A decrease in patients verbal numerical rating score (VNRS) by more than $40 \%$ pre and post block was considered as a positive block and the patient was scheduled for the intervention one week later. Patients' heels were classified into 2 groups, $(\mathrm{P})$ representing $\mathrm{PRF}, \mathrm{T})$ representing TRF.

\section{Intervention}

After sterilization and draping of the skin, local anesthesia was applied using $1 \mathrm{~mL}$ lidocaine $2 \%$ to anasthetize the site of the needle entrance (Fig. 1).

Using a Neurotherm 1000 Radio-Frequency Machine, Reusable Thermo-couple $5 \mathrm{~cm}$ length and Neurotherm radiofrequency cannula curved tip, $5 \mathrm{~cm}$ length and $10 \mathrm{~mm}$ active tip, the medial calcaneal nerve was localized via sensory stimulation (Figs. 2-3). Then $20 \mathrm{mg}$ methylprednisolone acetate and $1 \mathrm{~mL}$ of lidocaine $2 \%$ were injected followed by applying 90 seconds of TRF ablation at a set temperature of $800 \mathrm{C}$ in group $\mathrm{T}$ heels.

The other heel of the patient, Group P, had the same preparation and intervention except for applying PRF for 6 minutes at a set temperature of $420 \mathrm{C}$ to the medial calcaneal nerve instead of TRF for 90 seconds. Thus, all patients in the study had one side treated with the pulsed mode and the other side with the thermal mode. The patients were blinded regarding the RF mode used in their heels and were informed to expect gradual improvement in the next 3 weeks.

Patients were followed up using: Wake up numerical verbal rating score (W-NVRS), where 0 represents no pain and 10 represent the worst perceived pain when the patients woke up from bed (Fig. 4). Moreover, prolonged numerical verbal rating score (P-NVRS) for walking more than 15 minutes, and numerical rating score (NRS) for satisfaction were done (Figs. 5-6). The NRS marked 0 if the patients were completely unsatisfied and 10 for the most satisfaction. Analgesia was considered effective when pain scores dropped to $50 \%$ or more.

The patients were evaluated by the above scores before the procedure and after the end of one, 3, 6, 12, and 24 weeks from the intervention. The patients were asked to walk for 15 minutes just before they visited the clinic on the day of their evaluation and all the scores were filled in at the clinic.

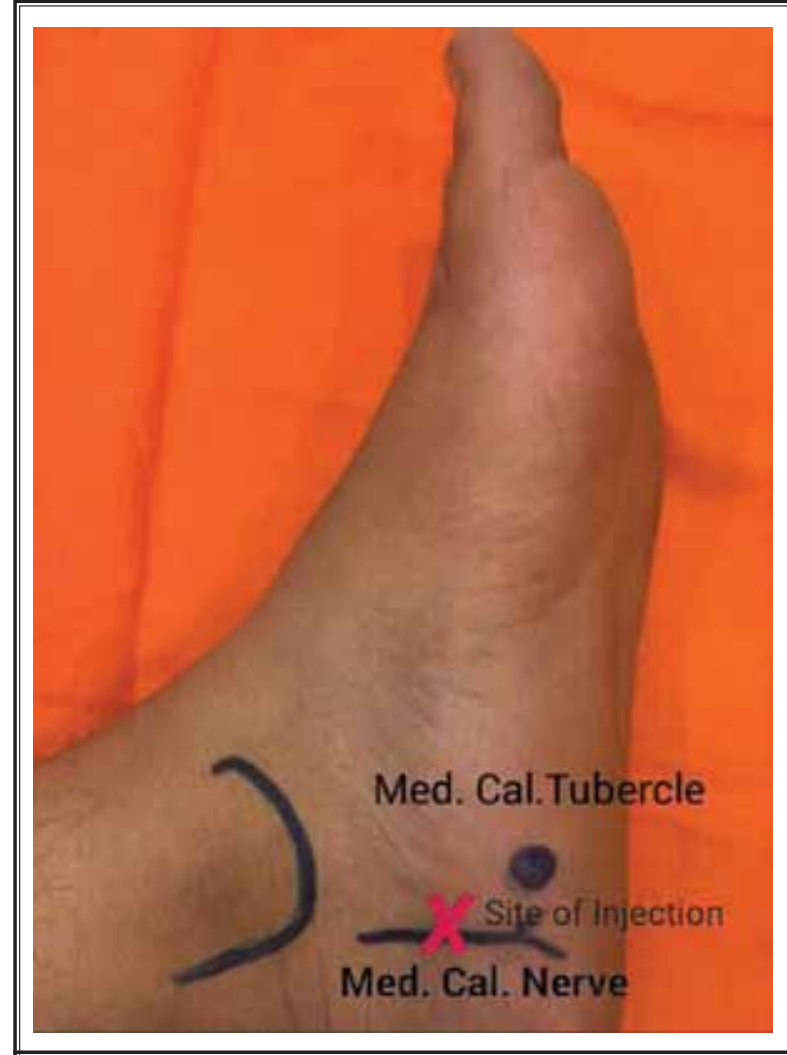

Fig. 1. A photo of the medial side of the left foot showing the site of injection.

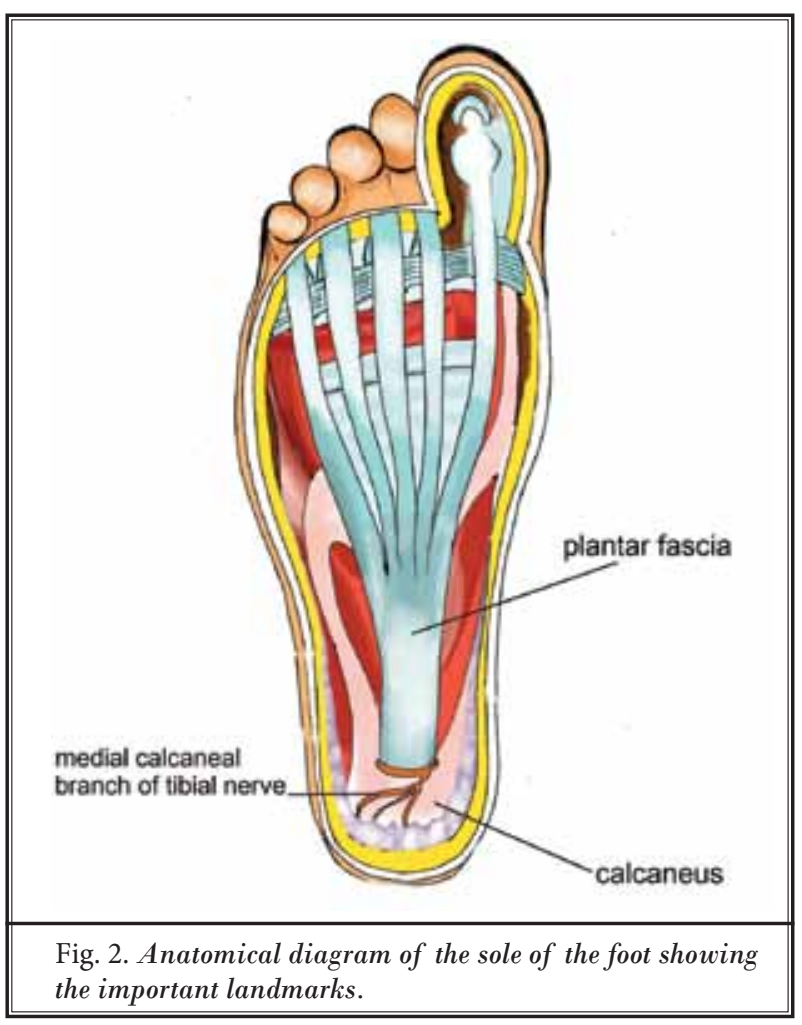
Fig. 2. Anatomical diagram of the sole of the foot showing
the important landmarks. 


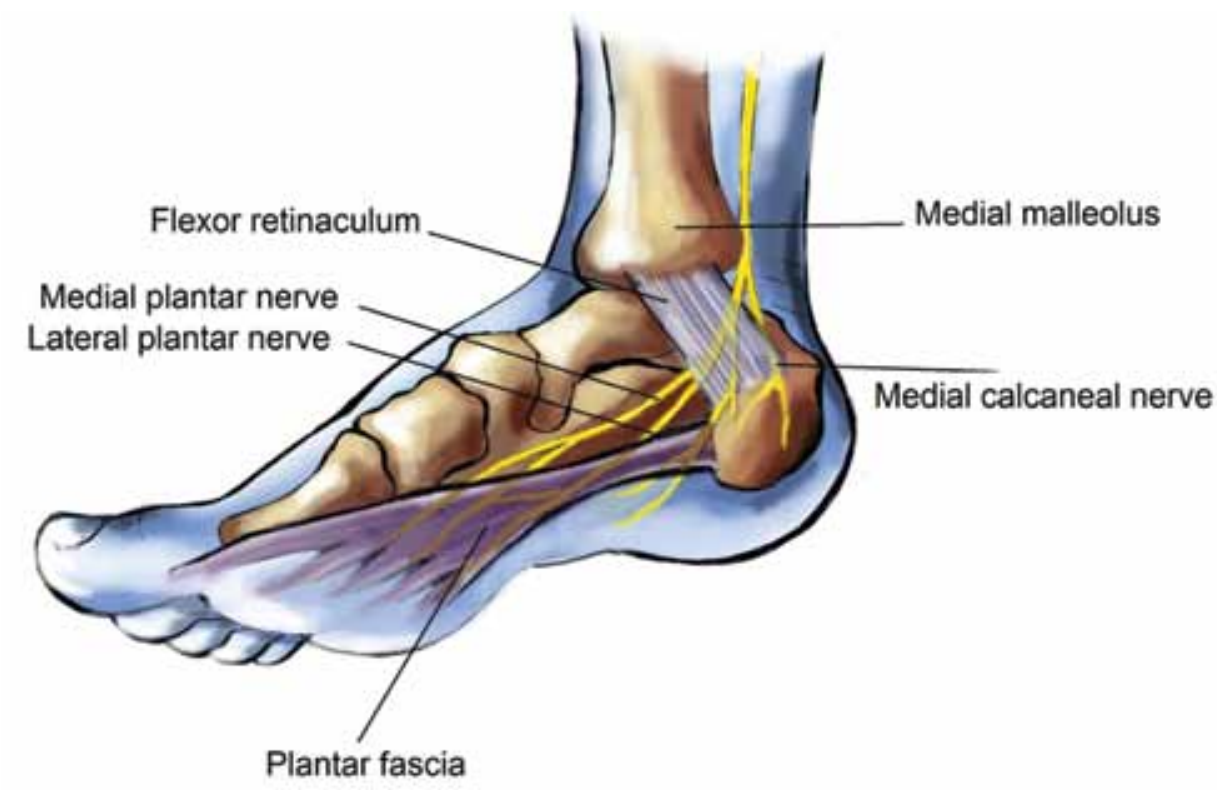

Fig. 3. Anatomical diagram of the right foot demonstrating planter fascia and its surrounding nerve supply.

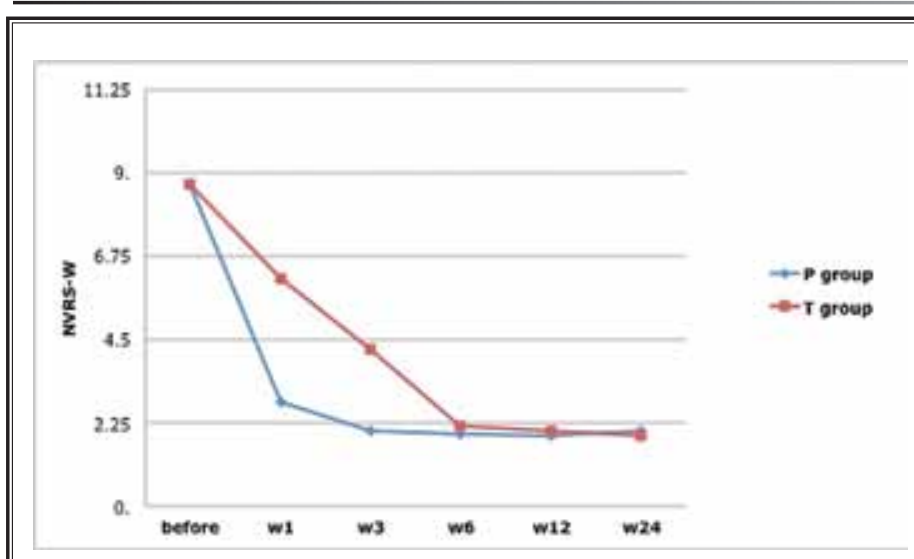

Fig. 4. Numerical verbal rating scale after wakingup in the 2 study groups (W-NVRS).

Data were collected and analyzed by SPSS version 21 (Chicago, USA). Data were expressed as mean, standard deviation, number, and percentage. Mann-Whitney was used to determine the significance for numeric variables. Chi square was used to determine significance for categorical variables. Paired T-test was used to determine significance between variables at different times in the same group. $P$ value was considered significant if less than 0.05 .

\section{Results}

Demographic data indicated that there were no significant differences found between the age and gender of the 2 study groups. Women represented $75 \%$ of participants, with mean age $46.90 \pm 12.38$ ranged between 29 and 68 years. The mean W-NVRS and P-NVRS scores for the 24 weeks are presented in Table 1 and 2 , respectively.

Baseline assessment before treatment showed that W-NVRS and P-NVRS scores were not significantly different between the 2 study groups. Patient W-NVRS and P-NVRS scores showed greater reduction in the first and third weeks in group $\mathrm{P}$ than in group $\mathrm{T}(P<0.000)$.

Satisfaction NRS is presented in Table 3 and it reveals significantly more satisfaction in group $P$ in the first and third weeks than in group $\mathrm{T}$.

Analgesia was effective (pain scores dropped $50 \%$ or more) in all patients $(100 \%)$ treated with PRF from the first week and throughout the whole study period. Only $10.0 \%$ of group $T$ patients had effective analgesia one week after the intervention. While, $50.0 \%$ of group $P$ patients achieved effective 
analgesia 3 weeks post treatment. By the end of the sixth week after treatment, all our patients in group $\mathrm{T}$ had reported effective analgesia.

\section{Discussion}

Using sensory nerve conduction study (NCS) for the medial calcaneal nerve, Chang et al (15) confirmed that medial calcaneal nerve neuropathy is associated with plantar fasciitis.

RF heat treatments have been used successfully in the field of medicine for over 30 years for various pain syndromes (3). Conventional RF applications are painful and carry the risk of neuritis. Additionally, they carry the possibility of unintentionally damaging some other nerve fibers. On the other hand, PRF is applied at low temperature $\left(42^{\circ} \mathrm{C}\right)$ and is less painful than conventional RF (16). PRF nerve lesioning is considered a safe intervention with minimal risks for neuritis, deafferentation pain, and neuroma formation $(9,12)$.

In the present study, treatment with PRF achieved effective analgesia after one week in all patients and continued throughout the whole study period (24 weeks). On the other hand, TRF achieved effective analgesia in only $10 \%$ of the patients after one week and in $50 \%$ of patients after 3 weeks.

To our knowledge, this is the first study to trace the patient progress in the first month after RF treatment for plantar fasciitis.

The faster onset of analgesic effect in the PRF group could be explained at least in part by its minimal risk for neuritis (12). However, another hypothesis could be that in PRF the probe tip temperature was never above $42^{\circ} \mathrm{C}$ so it did not cause thermal injury to the surrounding tissues, while in TRF, a temperature as high as $80^{\circ} \mathrm{C}$ could cause painful thermal insult to the tissues in its vicinity that could last for a week or more. In the current work, we used $10-\mathrm{mm}$ active tip cannulas that were provided by the manufacturer. On the other hand, using $5-\mathrm{mm}$ active tip cannulas may be associated with less discomfort.

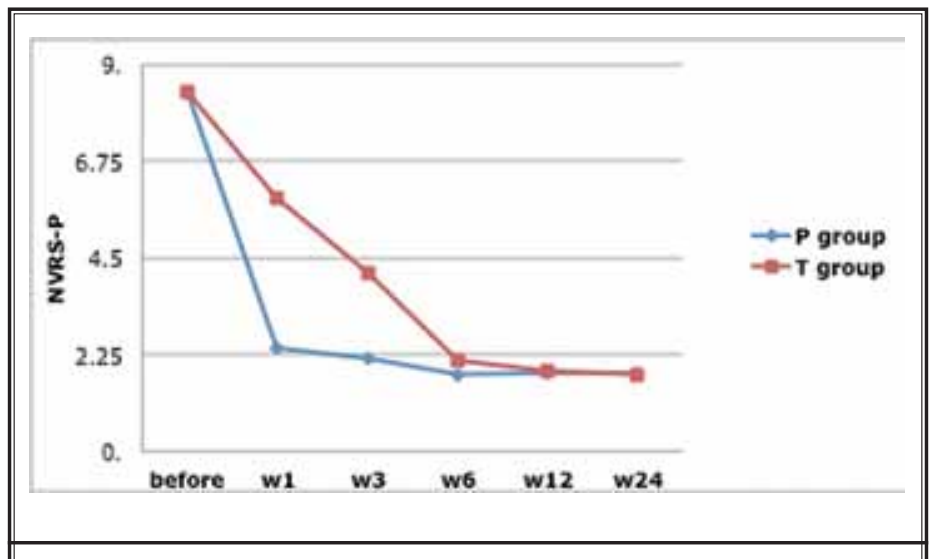

Fig. 5. Numerical verbal rating scale after prolonged walking in the 2 study groups ( $P$-NVRS).

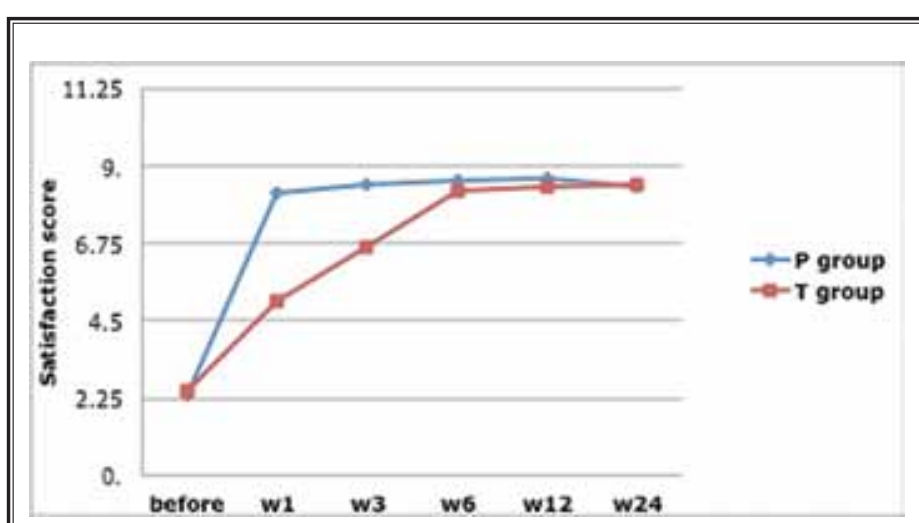

Fig. 6. Satisfaction through the follow-up visits in the 2 study groups.

Table 1. NVRS when waking up in study groups (W-NVRS).

\begin{tabular}{|l|c|c|c|}
\hline \multicolumn{1}{|c|}{ Item } & PRF & TRF & $\boldsymbol{P}$-value \\
\hline Before treatment & $8.70 \pm 0.14$ & $8.70 \pm 0.16$ & 1 \\
\hline Week 1 after treatment & $2.80 \pm 0.30^{*}$ & $6.15 \pm 0.33^{*}$ & $<0.001$ \\
\hline Week 3 after treatment & $2.05 \pm 0.27^{*}$ & $4.25 \pm 0.41^{*}$ & $<0.001$ \\
\hline Week 6 after treatment & $1.95 \pm 0.30$ & $2.15 \pm 0.28$ & 0.633 \\
\hline Week 12 after treatment & $1.90 \pm 0.29$ & $2.05 \pm 0.31$ & 0.730 \\
\hline Week 24 after treatment & $2.05 \pm 0.35$ & $1.90 \pm 0.28$ & 0.744 \\
\hline
\end{tabular}

${ }^{*}=P<0.05$ is considered significant

PRF: Pulsed Radiofrequency

TRF: Thermal Radiofrequency 
Table 2. NVRS at prolonged walking in the 2 study groups (P-NVRS).

\begin{tabular}{|l|c|c|c|}
\hline \multicolumn{1}{|c|}{ Item } & PRF & TRF & P-value \\
\hline Before treatment & $8.35 \pm 0.19$ & $8.35 \pm 0.18$ & 1 \\
\hline Week 1 after treatment & $2.40 \pm 0.31^{*}$ & $5.90 \pm 0.30^{*}$ & $<0.001$ \\
\hline Week 3 after treatment & $2.15 \pm 0.33^{*}$ & $4.15 \pm 0.42^{*}$ & $<0.001$ \\
\hline Week 6 after treatment & $1.80 \pm 0.27$ & $2.10 \pm 0.25$ & 0.427 \\
\hline Week 12 after treatment & $1.85 \pm 0.35$ & $1.90 \pm 0.25$ & 0.902 \\
\hline Week 24 after treatment & $1.85 \pm 0.30$ & $1.80 \pm 0.23$ & 0.897 \\
\hline
\end{tabular}

${ }^{*}=P<0.05$ is considered significant

PRF: Pulsed Radiofrequency

TRF: Thermal Radiofrequency

Table 3. Satisfaction score in study groups.

\begin{tabular}{|l|c|c|c|}
\hline \multicolumn{1}{|c|}{ Item } & PRF & TRF & P-value \\
\hline Before treatment & $2.35 \pm 0.18$ & $2.45 \pm 0.17$ & 0.690 \\
\hline Week 1 after treatment & $8.20 \pm 0.22^{*}$ & $5.10 \pm 0.30^{*}$ & $<0.001$ \\
\hline Week 3 after treatment & $8.45 \pm 0.21^{*}$ & $6.65 \pm 0.36^{*}$ & $<0.001$ \\
\hline Week 6 after treatment & $8.60 \pm 0.21$ & $8.30 \pm 0.23$ & 0.342 \\
\hline Week 12 after treatment & $8.65 \pm 0.16$ & $8.40 \pm 0.23$ & 0.390 \\
\hline Week 24 after treatment & $8.40 \pm 0.23$ & $8.45 \pm 0.23$ & 0.881 \\
\hline
\end{tabular}

${ }^{*}=P<0.05$ is considered significant

PRF: Pulsed Radiofrequency

TRF: Thermal Radiofrequency

Erken and colleagues (13) suggested that RF nerve ablation is an effective treatment for chronic heel pain associated with plantar fasciitis when it does not respond to other conservative treatment options. They used TRF with a maximum probe tip temperature of around $90^{\circ} \mathrm{C}$ and did a 2-year followup for their patients. However, their patients first follow-up visit was after one month and they did not comment on the progress of improvement before one month. Landsman and colleagues (17) demonstrated the efficacy of RF nerve ablation for the treatment of plantar fasciitis.

Liden and colleagues (14), in their retrospective study involving 22 patients ( $31 \mathrm{feet}$ ) with a history of prolonged moderate to severe heel pain associated with plantar fasciitis, concluded that RF nerve ablation to the sensory branch of the medial calcaneal nerve should be considered as an alternative to repetitive corticosteroid injections or open surgical intervention for the treatment of recalcitrant plantar heel pain.

Thapa and Ahuja described 2 case reports where PRF to the medial calcaneal nerve was effective in treat- ing refractory planter fasciitis pain (1).

The exact mechanisms of pain relief achieved by PRF are not fully understood. Changes in gene expression such as c-fos were among the proposed mechanisms (18). Hamann and coworkers (19) applied PRF to the sciatic nerve and dorsal root ganglion in a rat model and noted an upregulation of ATF3, an indicator of "cellular stress." This effect is seen only in smalldiameter C and A delta fibers which are responsible for transmission of pain. Thus, they hypothesized that the biological changes in the targeted nerve are due to an electro-mechanical effect and not due to a heat effect. They also reported that the main changes were detected in sensory fibers only and motor fibers were spared when using the electron microscope (19).

PRF nerve lesioning is a safe intervention (12). The risks of neuritis, deafferentation pain, and neuroma formation are minimal when compared to TRF (9).

\section{Limitations}

The current study had some limitations including non-randomization of the patients, small sample size, 
and long-term effectiveness of the procedure. These factors should be addressed in another study.

\section{Conclusion}

In conclusion, PRF on the medial calcaneal nerve is a safe and effective method for treatment of chronic planter fasciitis pain. The onset of effective analgesia can be achieved more rapidly with PRF compared to TRF on the same nerve. Further randomized trials are needed to confirm the therapeutic effect and to optimize the dose of RF needed.

\section{References}

1. Thapa D, Ahuja V. Combination of diagnostic medial calcaneal nerve block followed by pulsed radiofrequency for plantar fascitis pain: A new modality. Indian J Anaesth 2014; 58:183-185.

2. Buchbinder R. Clinical practice. Plantar fasciitis. N Engl J Med 2004; 350:2159-2166.

3. Cione JA, Cozzarelli J, Mullin CJ. A retrospective study of radiofrequency thermal lesioning for the treatment of neuritis of the medial calcaneal nerve and its terminal branches in chronic heel pain. J Foot Ankle Surg 2009; 48:142-147.

4. Tracey I. Imaging pain. $\mathrm{Br}$ J Anaesth 2008; 101:32-39.

5. Tu P, Bytomski JR. Diagnosis of heel pain. Am Fam Physician 2011; 84:909-916.

6. Roxas M. Plantar fasciitis: Diagnosis and therapeutic considerations. Altern Med Rev 2005;1 0:83-93.

7. Young CC, Rutherford DS, Niedfeldt MW. Treatment of plantar fasciitis. Am Fam Physician 2001; 63:467-474, 477-468.

8. Easley M. Wheeless Textbook For Orthopedics. Duke Orthopedics, USA, 2014.

9. Sluijter M, Racz G. Technical aspects of radiofrequency. Pain Pract 2002; 2:195-200.

10. Shah RV, Ericksen JJ, Lacerte M. Interventions in chronic pain management. 2. New frontiers: Invasive nonsurgical interventions. Arch Phys Med Rehabil 2003; 84:S39-S44

11. Cosman ER, Jr., Cosman ER, Sr. Electric and thermal field effects in tissue around radiofrequency electrodes. Pain Med 2005; 6:405-424.

12. Cahana A. Pulsed radiofrequency: A neurobiologic and clinical reality. Anesthesiology 2005; 103:1311; author reply 1313-1314.

13. Erken HY, Ayanoglu S, Akmaz I, Erler K, Kiral A. Prospective study of percutaneous radiofrequency nerve ablation for chronic plantar fasciitis. Foot Ankle Int 2014; 35:95-103.

14. Liden B, Simmons M, Landsman AS. A retrospective analysis of 22 patients treated with percutaneous radiofrequency nerve ablation for prolonged moderate to severe heel pain associated with plantar fasciitis. ] Foot Ankle Surg 2009; 48:642-647.
15. Chang CW, Wang YC, Hou WH, Lee XX, Chang KF. Medial calcaneal neuropathy is associated with plantar fasciitis. Clin Neurophysiol 2007; 118:119-123.

16. Karaman H, Tufek A, Kavak GO, Yildirim ZB, Celik F. Would pulsed radiofrequency applied to different anatomical regions have effective results for chronic pain treatment? J Pak Med Assoc 2011; 61:879-885.

17. Landsman AS, Catanese DJ, Wiener SN, Richie DH, Jr., Hanft JR. A prospective, randomized, double-blinded study with crossover to determine the efficacy of radio-frequency nerve ablation for the treatment of heel pain. J Am Podiatr Med Assoc 2013; 103:8-15.

18. Higuchi $Y$, Nashold BS, Jr., Sluijter M, Cosman E, Pearlstein RD. Exposure of the dorsal root ganglion in rats to pulsed radiofrequency currents activates dorsal horn lamina I and II neurons. Neurosurgery 2002; 50:850-855; discussion 856 .

19. Hamann W, Abou-Sherif S, Thompson $S$, Hall S. Pulsed radiofrequency applied to dorsal root ganglia causes a selective increase in $\mathrm{ATF}_{3}$ in small neurons. Eur ] Pain 2006; 10:171-176. 
